ISSN 1855-3966 (printed edn.), ISSN 1855-3974 (electronic edn.)

ARS MATHEMATICA CONTEMPORANEA 15 (2018) 39-51

https://doi.org/10.26493/1855-3974.1439.fdf

(Also available at http://amc-journal.eu)

\title{
Groups in which every non-nilpotent subgroup is self-normalizing
}

\author{
Costantino Delizia \\ University of Salerno, Italy \\ Urban Jezernik, Primož Moravec \\ University of Ljubljana, Slovenia \\ Chiara Nicotera \\ University of Salerno, Italy
}

Received 12 July 2017, accepted 29 August 2017, published online 2 November 2017

\begin{abstract}
We study the class of groups having the property that every non-nilpotent subgroup is equal to its normalizer. These groups are either soluble or perfect. We describe soluble groups and finite perfect groups with the above property. Furthermore, we give some structural information in the infinite perfect case.
\end{abstract}

Keywords: Normalizer, non-nilpotent subgroup, self-normalizing subgroup.

Math. Subj. Class.: 20E34, 20D15, 20E32

\section{Introduction}

A long standing problem posed by Y. Berkovich [3, Problem 9] is to study the finite $p$ groups in which every non-abelian subgroup contains its centralizer.

In [6], the finite $p$-groups which have maximal class or exponent $p$ and satisfy Berkovich's condition are characterized. Furthermore, the infinite supersoluble groups with the same condition are completely classified. Although it seems unlikely to be able to get a full classification of finite $p$-groups in which every non-abelian subgroup contains its centralizer, Berkovich's problem has been the starting point for a series of papers investigating finite and infinite groups in which every subgroup belongs to a certain family or it contains

E-mail addresses: cdelizia@unisa.it (Costantino Delizia), urban.jezernik@fmf.uni-lj.si (Urban Jezernik), primoz.moravec@fmf.uni-1j.si (Primož Moravec), cnicoter@unisa.it (Chiara Nicotera) 
its centralizer. For instance, in [7] and [9] locally finite or infinite supersoluble groups in which every non-cyclic subgroup contains its centralizer are described.

A more accessible version of Berkovich's problem has been proposed by P. Zalesskii, who asked to classify the finite groups in which every non-abelian subgroup equals its normalizer. This problem has been solved in [8].

In this paper we deal with the wider class $\mathcal{S}$ of groups in which every non-nilpotent subgroup equals to its normalizer. All nilpotent groups (and hence all finite $p$-groups) are in $\mathcal{S}$. It is also easy to see that groups in $\mathcal{S}$ are either soluble or perfect. Further obvious examples of groups in $\mathcal{S}$ include the minimal non-nilpotent groups (that is, non-nilpotent groups in which every proper subgroup is nilpotent) and groups in which every subgroup is self-normalizing. Finite minimal non-nilpotent groups are soluble, and their structure is well known (see [18, 9.1.9]). Infinite minimal non-nilpotent groups have been first studied in [14] (see also [4] for more recent results). These groups are either finitely generated or locally finite $p$-groups (Černikov groups or Heineken-Mohamed groups). Ol'shanskii and Rips (see [15]) showed that there exist finitely generated infinite simple groups all of whose proper non-trivial subgroups are cyclic of the same order (the so-called Tarski monsters). On the other side, groups whose non-trivial subgroups are self-normalizing are periodic and simple. Furthermore, in the locally finite case they are trivial or of prime order. Again, infinite examples are the Tarski $p$-groups.

We describe soluble groups lying in the class $\mathcal{S}$. It turns out that an infinite polycyclic group lies in the class $\mathcal{S}$ if and only if it is nilpotent (Proposition 3.3). We also prove that a non-periodic soluble group belongs to the class $\mathcal{S}$ if and only if it is nilpotent (Theorem 3.4). Moreover, a periodic soluble group which is not locally nilpotent lies in the class $\mathcal{S}$ if and only if it is a split extension of a nilpotent $p^{\prime}$-group by a cyclic $p$-group whose structure is described in Theorem 3.5. In particular, this result characterizes non-nilpotent soluble finite groups in the class $\mathcal{S}$. Furthermore, a locally nilpotent soluble group belongs to the class $\mathcal{S}$ if and only if it is either nilpotent or minimal non-nilpotent (Theorem 3.7).

In the last part of the paper we prove that a finite perfect group lies in the class $\mathcal{S}$ if and only if it is either isomorphic to the group $\operatorname{PSL}_{2}\left(2^{n}\right)$ where $2^{n}-1$ is a prime number, or to the group $\mathrm{SL}_{2}(5)$ (Theorem 4.8). Finally, we give some information on the structure of infinite perfect groups lying in the class $\mathcal{S}$.

Our notation is mostly standard (see for instance [3] and [18]). In particular, given any group $G$, we will denote by $Z(G)$ the center of $G$, by $Z^{\infty}(G)$ the hypercenter of $G$, by $\Phi(G)$ the Frattini subgroup of $G$, by $G^{\prime}$ the commutator subgroup of $G$, and, for all integers $i \geq 1$, by $\gamma_{i}(G)$ the $i$-th term of the lower central series of $G$.

\section{General properties of groups in $\mathcal{S}$}

It is very easy to prove that the class $\mathcal{S}$ is subgroup and quotient closed. Furthermore, non-nilpotent groups in $\mathcal{S}$ are not products of two proper normal subgroups.

Recall that a group $G$ is said to be perfect if it equals its commutator subgroup $G^{\prime}$. Clearly, if $G \in \mathcal{S}$ then $G$ is perfect or $G^{\prime}$ is nilpotent. Hence the groups in $\mathcal{S}$ are either perfect or soluble.

Suppose now that a cyclic group $\langle x\rangle$ acts on a group $H$ by means of an automorphism $x$. If a subgroup $L$ of $H$ is invariant with respect to $\langle x\rangle$, we will write $L \leq_{x} H$. Consider the induced map

$$
\rho_{x}: H \rightarrow H, \quad \rho_{x}(h)=[x, h]=h^{-x} h .
$$


Clearly, if $H$ is abelian then $\rho_{x}$ is a homomorphism. We will describe groups belonging to the class $\mathcal{S}$ based on the following property of $\rho_{x}$ :

$$
\forall K \leq_{x} H,\left(\exists n \geq 1: \rho_{x}^{n}(K)=1 \vee\left\langle\rho_{x}(K)\right\rangle=K\right) .
$$

Lemma 2.1. Let $x$ act on $H$ by means of an automorphism. Then for every $K \leq_{x} H$ we have $\left\langle\rho_{x}(K)\right\rangle K^{\prime}=\rho_{x}(K) K^{\prime}$.

Proof. Let $h_{1}, h_{2} \in K$. Then $\left[x, h_{1} h_{2}\right]=\left[x, h_{2}\right]\left[x, h_{1}\right]\left[x, h_{1}, h_{2}\right]$. It follows that $\rho_{x}\left(h_{1} h_{2}\right) \equiv \rho_{x}\left(h_{1}\right) \rho_{x}\left(h_{2}\right)\left(\bmod K^{\prime}\right)$.

The following easy observations are used in the sequel.

Lemma 2.2. Let $x$ act on $H$ by means of an automorphism.

1. The action of $x$ is fixed point free if and only if $\rho_{x}$ is injective.

2. If $\rho_{x}$ is injective and $H$ is abelian, then $(\star)$ implies that $\rho_{x}$ is an isomorphism.

3. If $\rho_{x}$ is injective (or surjective) and $H$ is finite, then $\rho_{x}$ satisfies $(\star)$.

Proof. (i) Note that $\rho_{x}$ is injective if and only if whenever $[x, h]=1$ it follows that $h=1$. This is precisely the same as $x$ acting fixed point freely on $H$.

(ii) Of course we can assume that $H$ is non-trivial. If $\rho_{x}$ is injective then there is no positive integer $n$ with the property that $\rho_{x}^{n}(K)=1$, and so $(\star)$ implies that $\left\langle\rho_{x}(H)\right\rangle=H$. If in addition $H$ is abelian then $\rho_{x}$ is a homomorphism, and hence $\left\langle\rho_{x}(H)\right\rangle=\rho_{x}(H)$. Therefore $\rho_{x}$ is an isomorphism.

(iii) If $H$ is assumed to be finite, then $\rho_{x}$ is injective if and only if it is surjective. In this case $\rho_{x}$ is bijective, and we have that $\rho_{x}(K)=K$ for all $K \leq_{x} H$. Thus $\rho_{x}$ satisfies $(\star)$.

Lemma 2.3. Let $G=\langle x\rangle H$, where $H$ is a nilpotent normal subgroup of $G$ generated by a set $Y$. Suppose that there exists $n \geq 1$ such that $\rho_{x}^{n}(y)=1$ for every $y \in Y$. Then $G$ is nilpotent.

Proof. By a theorem of Hall (see for instance [16, Theorem 2.27]) it suffices to show that $G / H^{\prime}$ is nilpotent. The group $H / H^{\prime}$ is generated by all $y H^{\prime}$ with $y \in Y$ and $\rho_{x}$ induces an endomorphism $\tau$ of $H / H^{\prime}$ such that $\tau^{n}\left(H / H^{\prime}\right)=1$. Now $G / H^{\prime}$ is nilpotent of class at most $n$ since $H / H^{\prime} \subseteq Z_{n}\left(G / H^{\prime}\right)$.

Lemma 2.4. Let $G=\langle x\rangle \ltimes H$ be a non-nilpotent group where $x$ has prime order $p$ and $H$ is nilpotent. Assume that $\rho_{x}$ has property $(\star)$ and suppose that there exists a subgroup $1 \neq K \leq_{x} H$ such that $\rho_{x}^{n}(K)=1$. Then $Z(G) \neq 1$.

Proof. As $\langle x\rangle \ltimes K$ is nilpotent by Lemma 2.3, it has a non-trivial center. Thus there exists an element $1 \neq h \in C_{K}(x)$. Now consider the group $\langle x\rangle \ltimes Z(H)$. By property $(\star)$, we either have $\rho_{x}(Z(H))=Z(H)$ or there is a positive integer $n$ such that $\rho_{x}^{n}(Z(H))=1$. In the latter case, we certainly have an element that belongs to $Z(H)$ and commutes with $x$, so that $Z(G) \neq 1$. Suppose now that $\rho_{x}(Z(H))=Z(H)$ holds. By property $(\star)$ we have

$$
\langle Z(H), h\rangle=\rho_{x}(\langle Z(H), h\rangle)=\rho_{x}(Z(H))=Z(H),
$$

and hence $h \in Z(H)$. Thus we again have $Z(G) \neq 1$. 
The following proposition shows how property $(\star)$ is tightly related to the class $\mathcal{S}$.

Proposition 2.5. Let $G=\langle x\rangle \ltimes H$ be a group in $\mathcal{S}$ with $x^{p}$ acting trivially on a nilpotent subgroup $H$ for some prime $p$. Then $\rho_{x}$ has property $(\star)$.

Proof. Let $K \leq_{x} H$, and suppose $\left\langle\rho_{x}(K)\right\rangle \subsetneq K$. Consider the subgroup

$$
L=\langle x\rangle \ltimes\left\langle\rho_{x}(K)\right\rangle K^{\prime}
$$

of $G$. As $K$ is nilpotent, it follows that $\left\langle\rho_{x}(K)\right\rangle K^{\prime} \subsetneq K$ (see for instance [16, Lemma 2.22]). Therefore $L$ is a proper normal subgroup of $\langle x\rangle \ltimes K$. Now, since $\langle x\rangle \ltimes K$ belongs to $\mathcal{S}$, it follows that its normal subgroup $L$ must be nilpotent, and so $\rho_{x}^{n}(K)=1$ for some positive integer $n$. Therefore $\rho_{x}$ has property $(\star)$.

Let $p$ be any prime number. An abelian group $A$ is said to be $p$-divisible if $A=p A$.

Lemma 2.6. Let $x$ be an automorphism of order $p$ of an abelian group A. If $\rho_{x}$ is surjective, then $A$ is p-divisible.

Proof. Consider $A$ as a $\mathbb{Z}[\langle x\rangle]$-module. In this sense, the operator $\rho_{x}$ corresponds to the element $1-x \in \mathbb{Z}[\langle x\rangle]$. We have $(1-x)^{p} \equiv 0$ modulo $p \mathbb{Z}[\langle x\rangle]$, and so the image of $\left(\rho_{x}\right)^{p}$ is a subgroup of $p \mathbb{Z}[\langle x\rangle] A=p A$. As $\rho_{x}$ is assumed to be surjective, it follows that $A=p A$.

Lemma 2.7. Let $G=\langle x\rangle \ltimes H$ be a periodic non-nilpotent group with $x^{p}=1$ for some prime $p$ and $H$ a nilpotent $p^{\prime}$-group. Assume that $\rho_{x}$ has property $(\star)$. Then every nonnilpotent subgroup $L \leq G$ is conjugate to a subgroup of the form $\langle x\rangle \ltimes K$ for some $K \leq_{x} H$.

Proof. Since $H$ is nilpotent, $L$ is not contained in $H$. It easily follows that $L$ contains an element of the form $x h$ for some $h \in H$. As $G$ is non-nilpotent, it follows from property (*) and Lemma 2.1 that

$$
h \in H=\left\langle\rho_{x}(H)\right\rangle \subseteq \rho_{x}(H) H^{\prime} .
$$

Hence we can write $h=\rho_{x}\left(h_{1}\right) h^{\prime}$ with $h_{1} \in H$ and $h^{\prime} \in H^{\prime}$. Thus $x h=x^{h_{1}} h^{\prime}$. After possibly replacing $L$ by $L^{h_{1}^{-1}}$, we can assume that $x h^{\prime} \in L$ for some $h^{\prime} \in H^{\prime}$. As $\rho_{x}$ has property $(\star)$, we have that either $\rho_{x}^{n}\left(H^{\prime}\right)=1$ for some positive integer $n$, or $\left\langle\rho_{x}\left(H^{\prime}\right)\right\rangle=H^{\prime}$.

In the first case $\langle x\rangle \ltimes H^{\prime}$ is nilpotent by Lemma 2.3. The subgroup $\left\langle x, h^{\prime}\right\rangle$ is finitely generated, periodic and nilpotent, therefore it is finite. Let $c$ be its nilpotency class, and let $p^{k}$ be the largest power of $p$ that divides $c$ !. Set $m$ to be a positive solution to the congruence system

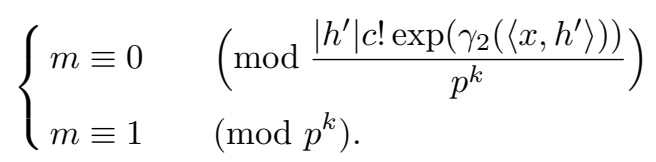

Thus $m(m-1)$ divisible by $c !, m$ is divisible by $\exp \left(\gamma_{2}\left(\left\langle x, h^{\prime}\right\rangle\right)\right)$ and by $\left|h^{\prime}\right|$, and $m$ is coprime to $p$. In particular, $\left(\begin{array}{c}m \\ i\end{array}\right)$ is divisible by $\exp \left(\gamma_{2}\left(\left\langle x, h^{\prime}\right\rangle\right)\right)$ for all $1 \leq i \leq c$. By the Hall-Petrescu formula (see for instance [3, Appendix 1]) we get

$$
\left(x h^{\prime}\right)^{m}=x^{m} h^{\prime m} g_{2}^{\left(\begin{array}{c}
m \\
2
\end{array}\right)} \ldots g_{c}^{\left(\begin{array}{c}
m \\
c
\end{array}\right)}
$$


with $g_{i} \in \gamma_{i}\left(\left\langle x, h^{\prime}\right\rangle\right)$. By the choice of $m$, it follows that $\left(x h^{\prime}\right)^{m}=x^{m}$. This element belongs to $L$, and since $x$ has $p$-power order, we conclude that $x \in L$.

Consider now the case when $\left\langle\rho_{x}\left(H^{\prime}\right)\right\rangle=H^{\prime}$. Thus $\langle x\rangle \ltimes H^{\prime}$ is non-nilpotent, and we can repeat the argument from above with $H$ replaced by $H^{\prime}$. Since $H$ is nilpotent, after finitely many steps we find a conjugate of $L$ that contains $x$. Replacing $L$ by this conjugate we can thus write $L=\langle x\rangle \ltimes K$ for $K=L \cap H \leq_{x} H$.

Proposition 2.8. Let $G=\langle x\rangle \ltimes H$ be a periodic group with $x^{p}$ acting trivially on $a$ nilpotent $p^{\prime}$-group $H$ for some prime $p$. Then $G \in \mathcal{S}$ if and only if $\rho_{x}$ has property $(\star)$.

Proof. If $G \in \mathcal{S}$, then $\rho_{x}$ has property $(\star)$ by Lemma 2.5 .

Conversely, assume now that $\rho_{x}$ has property $(\star)$. To prove that $G$ belongs to the class $\mathcal{S}$, take any non-nilpotent subgroup $L$ of $G$. By Lemma 2.7, we can assume that $L$ is of the form $L=\langle x\rangle \ltimes K$ for some $K \leq_{x} H$. Let us now show that $L$ is self-normalizing in $G$. To this end, take an element $x^{j} c \in N_{G}(L)$. Then $x^{x^{j} c}=x^{c}=x \rho_{x}(c)$, and so we must have $\rho_{x}(c) \in K$. Furthermore, we also have that $c=x^{-j}\left(x^{j} c\right) \in N_{H}(K)$. Note that $\left\langle\rho_{x}^{-1}(K) \cap N_{H}(K)\right\rangle \leq_{x} H$. It follows that

$$
N_{G}(L)=\langle x\rangle \ltimes\left\langle\rho_{x}^{-1}(K) \cap N_{H}(K)\right\rangle .
$$

Now, for any $h_{1}, h_{2} \in\left\langle\rho_{x}^{-1}(K) \cap N_{H}(K)\right\rangle$, we have that

$$
\rho_{x}\left(h_{1} h_{2}\right)=\rho_{x}\left(h_{2}\right) \rho_{x}\left(h_{1}\right)\left[x, h_{1}, h_{2}\right] \in K \cdot K \cdot\left[K, N_{H}(K)\right] \subseteq K .
$$

Therefore $\rho_{x}$ maps $\left\langle\rho_{x}^{-1}(K) \cap N_{H}(K)\right\rangle$ into $K$. Since $N_{G}(L)$ is not nilpotent, it follows from property $(\star)$ that

$$
\left\langle\rho_{x}^{-1}(K) \cap N_{H}(K)\right\rangle=\left\langle\rho_{x}\left(\left\langle\rho_{x}^{-1}(K) \cap N_{H}(K)\right\rangle\right)\right\rangle \subseteq K .
$$

This implies $N_{G}(L) \leq\langle x\rangle \ltimes K=L$, as required.

Remark 2.9. Assume $G=\langle x\rangle \ltimes H$ with $H$ an abelian finite group and $x$ acting so that $x^{p}$ acts trivially on $H$. Then $G \in \mathcal{S}$ if an only if $\rho_{x}$ has property $(\star)$, which in this case is equivalent by Lemma 2.2 to $x$ acting fixed point freely on $H$. Confer [8, Theorem 2.13].

Remark 2.10. Let $G$ be a periodic group in $\mathcal{S}$ with a splitting $\langle x\rangle \ltimes H$, and assume that $x^{p}=1$. It might not be the case that $x$ acts fixed point freely on $H$ (see Example 2.11). In such a situation, we have $C_{G}(x) \cap H \neq 1$. Therefore $C_{H}(x)$ is a subgroup of $H$ with $\rho_{x}\left(C_{H}(x)\right)=1$. It follows from Lemma 2.4 that $Z(G) \neq 1$. Now consider the factor group $\langle x\rangle \ltimes H / Z^{\infty}(G)$. This group is centerless, and so by the above argument $x$ must act fixed point freely on $H / Z^{\infty}(G)$.

Example 2.11. Let $x$ be the automorphism of the quaternion group $Q_{8}$ given by

$$
i \mapsto j, \quad j \mapsto-k
$$

This is an automorphism of order 3. Form the semidirect product $G=\langle x\rangle \ltimes Q_{8} \cong \mathrm{SL}_{2}(3)$. It is readily verified that all proper subgroups of $G$ are nilpotent, and so $G \in \mathcal{S}$. Note that $x$ has a non-trivial fixed point on $Q_{8}$, namely $(-1)^{x}=-1$. So we have $Z^{\infty}(G)=\langle-1\rangle$ with $G /\langle-1\rangle \cong \operatorname{Alt}(4)$, and $x$ acts fixed point freely on $Q_{8} /\langle-1\rangle \cong C_{2} \times C_{2}$. 


\section{Soluble groups in the class $\mathcal{S}$}

In this section, we inspect soluble groups in the class $\mathcal{S}$. It turns out that the non-periodic case is only possible if the group is nilpotent, whereas the periodic case is more subtle.

A Fitting group is one which equals its Fitting subgroup. Thus a Fitting group is a product of nilpotent normal subgroups, and therefore it is locally nilpotent. If $G \in \mathcal{S}$ and $F$ denote the Fitting subgroup of $G$, then clearly $G$ is a Fitting group or $F$ is nilpotent.

Lemma 3.1. Let $G \in \mathcal{S}$ be a soluble group, and $F$ the Fitting subgroup of $G$. Then the following hold:

1. $G^{\prime} \leq F$;

2. G is a Fitting group or $G / F$ has prime order;

3. if $G / G^{\prime}$ is finitely generated then $G$ is a Fitting group or $G / G^{\prime}$ is cyclic of primepower order.

4. if $G$ is non-nilpotent then the quotient group $G / G^{\prime}$ is a locally cyclic p-group for some prime $p$, and $G^{\prime}=\gamma_{3}(G)$.

Proof. Because $G^{\prime}$ is normal in $G$ and $G$ is assumed to belong to $\mathcal{S}$, it follows that $G^{\prime}$ must be nilpotent. Whence it is contained in the Fitting subgroup of $G$, proving (i).

Suppose now that $G$ is not a Fitting group. Since $G^{\prime} \leq F$ by (i), the group $G / F$ is abelian. Let $N / F$ be any proper subgroup of $G / F$. Then $N$ is a proper normal subgroup of $G$, so $N$ is nilpotent. Thus $N \leq F$. Therefore $G / F$ has no proper non-trivial subgroups, so it has prime order. Hence (ii) is proved.

In order to prove (iii), suppose that $G / G^{\prime}$ is finitely generated and $G$ is not a Fitting group. Let $M_{1} / G^{\prime}$ and $M_{2} / G^{\prime}$ be maximal subgroups of $G / G^{\prime}$. Since $M_{1}$ and $M_{2}$ are proper normal subgroups of $G$, they are nilpotent and hence contained in $F$. If $M_{1} \neq M_{2}$ it follows that $G=M_{1} M_{2} \leq F$, a contradiction. This means that the finitely generated group $G / G^{\prime}$ has an unique maximal subgroup. Therefore $G / G^{\prime}$ is cyclic of prime-power order.

Finally assume $G$ is non-nilpotent, and let $H$ and $K$ be proper normal subgroups of $G$. Then $H$ and $K$ are nilpotent, hence $H K$ is nilpotent and so $H K \neq G$. Thus (iv) follows by [14, Theorem 2.12].

Proposition 3.2. Let $G=\langle x\rangle \ltimes A$ where $A$ is non-periodic abelian, $x$ acts fixed point freely on $A$ and $x^{p}$ acts trivially on $A$ for some prime $p$. Then $G$ does not lie in the class $\mathcal{S}$.

Proof. Consider $W=\langle x, y\rangle$ with $y \in A$ and of infinite order. The group $W \cap A$ is abelian and finitely generated, since there are only finitely many conjugates of $y$ in $W$. So also the torsion subgroup $T$ of $W \cap A$ has finite order, $m$ say. Then $(W \cap A)^{m}$ is finitely generated and torsion-free. By Lemma 2.6, the map $\rho_{x}$ restriced to $(W \cap A)^{m}$ is not surjective. Since $x$ acts fixed point freely on $A$ and therefore also on $(W \cap A)^{m}$, the group $\langle x\rangle \ltimes(W \cap A)^{m}$ is not nilpotent, and so $\rho_{x}^{n}\left((W \cap A)^{m}\right) \neq 1$ for all integers $n$. Whence $\rho_{x}$ restricted to $(W \cap A)^{m}$ does not have property $(\star)$. It follows from Proposition 2.5 that $G$ does not belong to the class $\mathcal{S}$.

Recall that a group is called just-infinite if it is infinite, but each of its proper quotients is finite. In particular a just-infinite group has no non-trivial finite normal subgroups. The Baer radical of a group $G$ is the subgroup generated by all the cyclic subnormal subgroups 
of $G$. It has been proved in [19, Theorem 2] that if $G$ is a just-infinite group with non-trivial Baer radical $A$ then $A$ is free abelian of finite rank and $C_{G}(A)=A$.

Proposition 3.3. Every infinite polycyclic group in $\mathcal{S}$ is nilpotent.

Proof. Assume by a contradiction that the result is false, and let $G \in \mathcal{S}$ be an infinite polycyclic group which is non-nilpotent. Then Lemma 3.1 (iii) ensures that $G / G^{\prime}$ is cyclic of prime-power order. It easily follows (see for instance [14, Corollary 2.11]) that $G^{\prime}=$ $\gamma_{3}(G)$.

Let consider the set $\mathcal{F}$ of all normal subgroups $N$ of $G$ such that $G / N$ is infinite and non-nilpotent. Thus $\mathcal{F}$ is not empty, as it contains the trivial subgroup. Since $G$ satisfies the maximal condition on subgroups, there exists a maximal element $M \in \mathcal{F}$. Hence $G / M$ is a non-nilpotent infinite polycyclic group in $\mathcal{S}$. Moreover, if $G / N$ is any infinite quotient of $G / M$, then the maximality of $M$ implies that either $N=M$ or $G / N$ is nilpotent. Suppose the latter holds. Then there exists a positive integer $t$ such that $\gamma_{t}(G) \leq N$. Thus $G^{\prime} \leq N$, a contradiction since $G / G^{\prime}$ is finite. Therefore, at expense of replacing $G$ by $G / M$, we may assume that $G$ is just-infinite.

Let $F$ denote the Fitting subgroup of $G$. Thus $F$ coincides with the Baer radical of $G$. Then $F$ is free abelian of finite rank by [19, Theorem 2]. Furthermore, by Lemma 3.1 (ii) we may assume that $G / F$ has prime order $p$. Hence, for all $x \in G \backslash F$ we can write $G=\langle x\rangle F$ with $x \notin F$ and $x^{p} \in F$. We claim that $F / C_{F}(x)$ is torsion-free. Indeed, suppose $a \in F \backslash C_{F}(x)$ with $a^{n} \in C_{F}(x)$ for some integer $n$. Thus $\left(a^{n}\right)^{x}=a^{n}$. Since $F$ is abelian it follows that $\left(a^{x} a^{-1}\right)^{n}=1$. Hence $n=0$ since $F$ is normal and torsion-free. Therefore $F / C_{F}(x)$ is torsion-free, as claimed. Thus $G / C_{F}(x)$ is infinite, which implies that $C_{F}(x)=1$. Hence $x$ acts fixed point freely on $F$. By Proposition 3.2, the group $G$ does not belong to the class $\mathcal{S}$, our final contradiction.

Theorem 3.4. A non-periodic soluble group belongs to the class $\mathcal{S}$ if and only if it is nilpotent.

Proof. Clearly, if $G$ is nilpotent then $G \in \mathcal{S}$.

Conversely, let $G \in \mathcal{S}$ be a non-periodic soluble group, and assume that $G$ is nonnilpotent. Let us first prove that in this case $G$ is locally nilpotent.

By assumption, there exists a finitely generated infinite subgroup of $G$, say $H$. Let $K$ be any finitely generated subgroup of $G$. Then the subgroup $J=\langle K, H\rangle$ is finitely generated and infinite. Assume that $J$ is non-nilpotent. Then its Fitting subgroup $F$ is nilpotent, and $J / F$ has prime order by Lemma 3.1 (ii). Hence $J$ is polycyclic, a contradiction by Proposition 3.3. Thus $J$ is nilpotent, and so is $K$. Therefore $G$ is locally nilpotent. Then by Lemma 3.1 (iv) the quotient group $G / G^{\prime}$ is a $p$-group for some prime $p$.

Assume now that $G$ is torsion-free. Since $G^{\prime}$ is nilpotent and $G / G^{\prime}$ is periodic, it follows by [17, Lemma 6.33] that $G$ is nilpotent, again a contradiction.

We are left with the case when the torsion subgroup $T$ of $G$ is non-trivial. Since $G / T$ is a torsion-free soluble group belonging to the class $\mathcal{S}$, it is nilpotent by the above. As $G / G^{\prime}$ is a $p$-group it follows that the quotient group $(G / T) /(G / T)^{\prime}$ is a $p$-group. Hence $G / T$ is a $p$-group (see for instance $[18,5.2 .6]$ ). Therefore $G$ is periodic, our final contradiction.

Next two results give a complete description of periodic soluble groups in $\mathcal{S}$. In particular, our next theorem characterizes finite soluble non-nilpotent groups in $\mathcal{S}$. 
Theorem 3.5. Let $G$ be a periodic soluble group, and assume that $G$ is not locally nilpotent. Then $G \in \mathcal{S}$ if and only if $G$ splits as $G=\langle x\rangle \ltimes H$, where $\langle x\rangle$ is a p-group for some prime $p, H$ is a nilpotent $p^{\prime}$-group, $x^{p}$ acts trivially on $H$ and $\rho_{x}$ has property $(\star)$.

Proof. If $G$ splits according to the above statement, then it follows from Propostion 2.8 that $G$ belongs to the class $\mathcal{S}$.

Assume now that $G \in \mathcal{S}$. Let $x$ be an element of $G$ that does not belong to the Fitting subgroup $F$. Then $x^{p} \in F$ for some prime $p$, by Lemma 3.1. After possibly replacing $x$ by one of its powers, we can assume that the order of $x$ is a power $p^{m}$ of $p$. As $G$ is not a Fitting group, we have that $F$ is nilpotent. Hence $F$ is a product of its Sylow subgroups, say $F=\prod_{q} S_{q}$. Note that there is at least one prime $q \neq p$ involved: otherwise $F$ is a $p$-group, hence $G$ is a $p$-group, but this yields that $G$ is locally nilpotent since it is locally finite, a contradiction. The Sylow subgroups of $F$ are all characteristic in $F$, so the conjugation action of $x$ preserves them. Therefore $x$ acts component-wise on $F$.

Note that $x^{p} \in S_{p}$. Consider the subgroup $P=\left\langle x, S_{p}\right\rangle$. Clearly, $P$ is a $p$-group. Assume that $P \neq\langle x\rangle$, and choose an element $y \in P \backslash\langle x\rangle$. Set

$$
J=\left\langle x, y, y^{x}, y^{x^{2}}, \ldots, y^{x^{p^{m}-1}}\right\rangle .
$$

Since $J$ is a finite $p$-group, and $\langle x\rangle \neq J$, it follows that

$$
\langle x\rangle \subsetneq N_{J}(\langle x\rangle) \subseteq N_{P}(\langle x\rangle)
$$

Hence there exists an element $z \in N_{P}(\langle x\rangle) \backslash\langle x\rangle$. Thus $G$ contains the subgroup $\langle x\rangle \ltimes$ $\prod_{q \neq p} S_{q}$ that is not self-normalizing. By assumption, this subgroup must be nilpotent. Since $x$ acts component-wise on $F$, it follows that $G$ itself should be nilpotent. This is a contradiction, from which it follows that $P=\langle x\rangle$, and so $S_{p}=\left\langle x^{p}\right\rangle$. This immediately implies that $G$ splits as $G=\langle x\rangle \ltimes H$ with $x$ acting component-wise on $H=\prod_{q \neq p} S_{q}$.

Since $x^{p} \in F$, it commutes with all the $q$-Sylow subgroups of $F$ for $q \neq p$. As the $p$-Sylow subgroup $S_{p}$ is cyclic, it follows that $x^{p} \in Z(G)$.

Finally, let $K \leq_{x} H$ with $\rho_{x}^{n}(K) \neq 1$ for all integers $n$. Therefore the group $\langle x\rangle \ltimes K$ is non-nilpotent. Consider the group $\langle x\rangle \ltimes\left\langle\rho_{x}(K)\right\rangle K^{\prime}$. It is a normal subgroup of $\langle x\rangle \ltimes K$, so it must either be equal to $\langle x\rangle \ltimes K$, or else it is nilpotent. The latter case implies that the group $\left\langle x, \rho_{x}(K)\right\rangle$ is nilpotent, which gives that $\rho_{x}^{n}(K)=1$ for some $n$, a contradiction. Hence we get that $\left\langle\rho_{x}(K)\right\rangle K^{\prime}=K$, and since $K$ is nilpotent, it follows that $\left\langle\rho_{x}(K)\right\rangle=K$. Thus $\rho_{x}$ has property $(\star)$.

Corollary 3.6. Let $n>2$. The dihedral group Dih $(n)$ of order $2 n$ belongs to $\mathcal{S}$ if and only if either $n$ is a power of 2 or $n$ is odd.

Theorem 3.7. A locally nilpotent soluble group lies in the class $\mathcal{S}$ if and only if it is either nilpotent or minimal non-nilpotent.

Proof. Clearly, nilpotent and minimal non-nilpotent groups belong to the class $\mathcal{S}$.

Let $G \in \mathcal{S}$ be a periodic soluble group which is locally nilpotent, and assume that $G$ is non-nilpotent. We will prove that $G$ is minimal non-nilpotent. For the sake of contradiction, assume that there exists a proper non-nilpotent subgroup $H$ of $G$. Let $B$ be the last term of the derived series of $G$ which is not contained in $H$. Then $H B$ has the proper nonnilpotent subgroup $H$. Hence without loss of generality we may assume that $G=H B$. 
Put $L=B \cap H$. Then $B^{\prime} \leq L$, so $L$ is normal in $B$. Obviously $L$ is normal in $H$, thus $L$ is normal in $G$. The normal series $L<B<G$ can be refined to a (general) principal series of $G$ (see for instance $[18,12.4 .1]$ ). Let $W / V$ be any factor of this principal series with $W \leq B$. As $G$ is locally nilpotent, the principal factor $W / V$ is central (see for instance $[18,12.1 .6])$. Hence $[W, G] \leq V$. This implies that $W \leq N_{G}(H V)=H V$. Therefore

$$
W=W \cap H V=(W \cap H) V \leq L V=V .
$$

This means $L=B$, a contradiction, and that proves our result.

Corollary 3.8. A locally nilpotent soluble group lying in the class $\mathcal{S}$ is nilpotent or a $p$ group for some prime $p$.

Proof. Let $G \in \mathcal{S}$ be a locally nilpotent soluble periodic group, and assume that $G$ is non-nilpotent. Then by Theorem 3.7 the group $G$ is minimal non-nilpotent, and the result follows by [14, Lemma 4.2].

\section{Perfect groups in the class $\mathcal{S}$}

Lemma 4.1. Let $G \in \mathcal{S}$ be a finite perfect group, and let $F$ denote its Fitting subgroup. Then $G / F$ is a non-abelian simple group.

Proof. If there is a proper normal subgroup $F \leq M<G$, then $M$ must be nilpotent since $G \in \mathcal{S}$, and so $M=F$. Thus $G / F$ is simple. As $G$ is also assumed to be perfect, $G / F$ is non-abelian.

We first classify the finite simple groups in $\mathcal{S}$. This is done with the help of the following lemma.

Lemma 4.2. Let $G$ be a finite simple group. Then $G$ belongs to $\mathcal{S}$ if and only if all of its maximal subgroups belong to $\mathcal{S}$.

Proof. Assume that all maximal subgroups of a finite simple group $G$ belong to $\mathcal{S}$, and let $H$ be a non-nilpotent proper subgroup of $G$. As $G$ is simple, we have $N_{G}(H)<G$, and so there is a maximal subgroup $M \leq G$ with $N_{G}(H) \leq M$. Since $M$ belongs to $\mathcal{S}$, it follows that $N_{G}(H)=N_{M}(H)=H$, as required.

Lemma 4.3. The group $\operatorname{PSL}_{2}(q)$ belongs to $\mathcal{S}$ if and only if $q=2^{n}$ with $q-1$ a prime, or $q \leq 5$.

Proof. Suppose that $\operatorname{PSL}_{2}(q)$ belongs to $\mathcal{S}$ with $q>5$, and assume first that $q$ is odd. This group contains dihedral subgroups of orders $(q-1) / 2$ and $(q+1) / 2$ by [10]. Unless $q=7$, at least one of these does not belong to $\mathcal{S}$ by Corollary 3.6. Note that $\mathrm{PSL}_{2}(7)$ has a subgroup isomorphic to $\operatorname{Sym}(4)$, so it does not belong to $\mathcal{S}$. Whence we can assume that $q=2^{n}$ for some $n \geq 3$. Now $\operatorname{PSL}_{2}(q)$ contains a diagonal torus of order $q-1$ acting fixed point freely on the unipotent subgroup of order $q$. It follows from Lemma 3.1 that the torus must be simple, and so $q-1$ is either trivial or a prime, as required. Finally, it follows from [8, Theorem 2.17] that such groups indeed belong to $\mathcal{S}$.

Proposition 4.4. A finite non-abelian simple group belongs to $\mathcal{S}$ if and only if it is isomorphic to $\mathrm{PSL}_{2}\left(2^{n}\right)$, where $2^{n}-1$ is a prime. 
Proof. We reduce the situation to the case of Lemma 4.3 by using Lemma 4.2.

- Alternating groups. It may be verified readily that $\operatorname{Alt}(n)$ belongs to $\mathcal{S}$ if and only $n=5$, since $\operatorname{Sym}(4)$ is contained in $\operatorname{Alt}(n)$ for every $n \geq 6$.

- Linear groups $\operatorname{PSL}_{n}(q)$. If $n=2$, this case is covered by Lemma 4.3. If $n \geq 3$, then there is a block embedding of $\mathrm{SL}_{2}(q)$ into $\operatorname{PSL}_{n}(q)$. The image of this subgroup is normalized by the class of a diagonal matrix of the form $\operatorname{diag}(\alpha, \beta, \gamma, 1, \ldots, 1)$. As long as $\alpha \neq \beta$, this diagonal matrix does not belong to the image of the embedding of $\mathrm{SL}_{2}(q)$, and so $\mathrm{PSL}_{n}(q)$ does not belong to $\mathcal{S}$. The only exceptional case is when $\left|\mathbb{F}_{q}^{\times}\right|=1$, i.e., $q=2$, in which case either $n=3$ or $\operatorname{PSL}_{n}(2)$ contains $\mathrm{SL}_{3}(2)$ via a block diagonal embedding. Both of these groups quotient onto $\mathrm{PSL}_{3}(2) \cong \mathrm{PSL}_{2}(7)$, which does not belong to $\mathcal{S}$.

- Symplectic groups $\operatorname{PSp}_{2 n}(q)$. If $n=1$, then $\operatorname{PSp}_{2}(q) \cong \operatorname{PSL}_{2}(q)$ and this is covered above. Now let $n>1$. Letting $W$ be a maximal isotropic subspace of the $2 n$ dimensional vector space on which $\operatorname{Sp}_{2 n}(q)$ acts, the stabilizer of the decomposition $W \oplus W^{\perp}$ is $\mathrm{GL}_{n}(q) \rtimes C_{2}$, and so $\mathrm{PSp}_{2 n}(q)$ contains $\mathrm{PGL}_{n}(q) \rtimes C_{2}$. Therefore these groups do not belong to $\mathcal{S}$.

- Unitary groups and orthogonal groups. Their associated root systems contain a subsystem of type $A_{2}$, and so they contain subgroups that are isomorphic to either $\mathrm{SL}_{3}(q)$ or $\mathrm{PSL}_{3}(q)$. None of these belong to $\mathcal{S}$ by above. See [2].

- Exceptional Chevalley groups. We have an inclusion

$$
G_{2}(q) \subset F_{4}(q) \subset E_{6}(q) \subset E_{7}(q) \subset E_{8}(q),
$$

and the list of maximal subgroups of $G_{2}(q)$ in [20, p. 127] shows that $G_{2}(q)$, and hence all of the above groups, does not belong to $\mathcal{S}$.

- Steinberg groups ${ }^{2} E_{6}\left(q^{2}\right)$ and ${ }^{3} D_{4}\left(q^{3}\right)$. By [20, Theorem 4.3], the group ${ }^{3} D_{4}\left(q^{3}\right)$ has a maximal subgroup which is isomorphic to $G_{2}\left(q^{3}\right)$, hence it is not in $\mathcal{S}$ by the above. Similarly, $F_{4}\left(q^{2}\right)$ embeds into ${ }^{2} E_{6}\left(q^{2}\right)$ by [20, p. 173], hence the latter is not in $\mathcal{S}$.

- Suzuki groups $\mathrm{Sz}(q)$. By [20, Theorem 4.1], these contain Frobenius groups $C_{q+\sqrt{2 q}+1} \ltimes C_{4}$ whose Fitting subgroups are of index 4 . Such groups do not belong to $\mathcal{S}$ by Lemma 3.1.

- Ree families. By [20, Theorem 4.2], $2 \times \mathrm{PSL}_{3}(2 n+1)$ is a maximal subgroup of ${ }^{2} G_{2}\left(3^{2 n+1}\right)$, and $\mathrm{Sz}\left(2^{2 n+1}\right)<2$ is a maximal subgroup of ${ }^{2} F_{4}\left(2^{2 n+1}\right)$ by [20, Theorem 4.5]. For the remaining case, ${ }^{2} F_{4}(2)^{\prime}$, we use ATLAS [5] to conclude that this group contains $\operatorname{Sym}(6)$.

- Sporadic groups. Inspection of ATLAS reveals that each of 26 sporadic groups has a maximal subgroup which is clearly not in $\mathcal{S}$.

To deal with perfect finite groups in $\mathcal{S}$, we make use of the theory of Schur covering groups. In particular, we will require the following.

Theorem 4.5 (Hauptsatz 23.5 of [11]). Let $G$ be a finite group and suppose there is an extension

$$
1 \rightarrow K \rightarrow E \rightarrow G \rightarrow 1
$$

with the property that $K \leq Z(E) \cap E^{\prime}$. Then $K$ embeds into the Schur multiplier $M(G)$. 
Proposition 4.6. Let $G \in \mathcal{S}$ be a perfect non-simple finite group, and let $F$ denote its Fitting subgroup. Assume that the group $G / F$ contains two elements a and $b$ of distinct prime orders with the additional property that $N_{G / F}(\langle a\rangle) \supsetneq\langle a\rangle$ and $N_{G / F}(\langle b\rangle) \supsetneq\langle b\rangle$. Then the group $S_{p} / \Phi\left(S_{p}\right)$ embeds into the Schur multiplier $M(G / F)$, for every $p$-Sylow subgroup $S_{p}$ of $F$.

Remark 4.7. It is easy to find such elements $a, b$ for the simple groups $\mathrm{PSL}_{2}\left(2^{n}\right)$ that appear in Proposition 4.4. One can take $a$ to be an involution (normalized by the Sylow 2 -subgroup of order $2^{n}$ ) and $b$ a diagonal matrix of order $q-1$ (normalized by the class of the flip $\left.\left(\begin{array}{ll}0 & 1 \\ 1 & 0\end{array}\right)\right)$.

Proof. The group $F$ is nilpotent, so we can write $F=\prod_{q} S_{q}$ where $S_{q}$ is a $q$-group. Now fix a prime $p$ and consider $G_{1}=G / \prod_{q \neq p} S_{q}$. The Fitting subgroup of $G_{1}$ is isomorphic to $S_{p}$. Further, consider the group $G_{2}=G_{1} / \Phi\left(S_{p}\right)$. The Fitting subgroup $F_{2}$ of $G_{2}$ is an elementary abelian $p$-group, and $G_{2}$ belongs to the class $\mathcal{S}$.

Write $S=G / F$. Then $S$ is simple by Lemma 4.1. The group $G_{2}$ acts on its subgroup $F_{2}$ by conjugation. There is thus an induced homomorphism $G_{2} \rightarrow \operatorname{Aut}\left(F_{2}\right)$. This homomorphism factors through $F_{2}$, so we get a homomorphism

$$
\psi: S \cong G_{2} / F_{2} \rightarrow \operatorname{Aut}\left(F_{2}\right) .
$$

As $S$ is a simple group, we have that either $\psi$ is injective or trivial. Let us show that $\psi$ must be trivial.

For the sake of contradiction, assume that ker $\psi=1$. Since $F_{2}$ is a $p$-group, at least one of the elements $a, b$ from the statement of the proposition has order coprime to $p$. Without loss of generality, assume this element is $a$. Now consider the group $H=\left\langle a, F_{2}\right\rangle \leq G_{2}$. By our assumption on the element $a$, the group $H$ is not self-normalized in $G_{2}$. But it is also not nilpotent. Indeed, the element $a$ acts nontrivially on $F_{2}$ because $\psi$ is an embedding of $S$ into $\operatorname{Aut}\left(F_{2}\right)$. The order of $a$ is coprime to $p$, so $\psi$ restricted to $\langle a\rangle$ is a completely reducible representation of $\langle a\rangle$ on the $G F(p)$-vector space $F_{2}$. This representation splits as a sum of 1-dimensional representations, and so $a$ is a diagonalizable element in the image of $\psi$. Being non-trivial, we can not have that $\psi(a)-I$ is a nilpotent matrix, and so the group $H$ can not be nilpotent. This leads to a contradiciton with the fact that $G_{2} \in \mathcal{S}$.

We therefore have that $\psi$ is trivial, and so $S$ acts trivially on $F_{2}$. This means that $F_{2}$ is central in $G_{2}$. Since $G_{2}$ is also assumed to be perfect, the extension $1 \rightarrow F_{2} \rightarrow$ $G_{2} \rightarrow S \rightarrow 1$ has the property that $F_{2} \leq Z\left(G_{2}\right) \cap G_{2}^{\prime}$. It follows from Theorem 4.5 that $F_{2} \cong S_{p} / \Phi\left(S_{p}\right)$ embeds into $M(S)$.

Our next result, together with Proposition 4.4, gives a complete classification of all finite perfect groups in $\mathcal{S}$.

Theorem 4.8. A finite perfect group $G$ belongs to the class $\mathcal{S}$ if and only if it is either isomorphic to $\mathrm{PSL}_{2}\left(2^{n}\right)$ where $2^{n}-1$ is a prime, or to $\mathrm{SL}_{2}(5)$.

Proof. The Fitting quotient of $G$ is a finite simple group belonging to $\mathcal{S}$, so it must be one of the PSL's appearing in Proposition 4.4. Note that we have $M\left(\mathrm{PSL}_{2}(4)\right) \cong C_{2}$ and all the other PSL's have trivial Schur multipliers. Therefore the only possibility for a non-simple perfect group $G$ in $\mathcal{S}$ is a group whose Fitting quotient is $\operatorname{PSL}_{2}(4)$. Such a group must have $F$ a 2-group with cyclic Frattini quotient, so $F$ itself is cyclic. But now as $G / F$ acts trivially on the Frattini quotient of $F$, it follows that the image of the 
homomorphism $G / F \rightarrow \operatorname{Aut}(F)$ is a $p$-group [12, Exercise 4.4]. Since $G / F$ is a nonabelian simple group, this implies that $G / F$ must act trivially even on $F$. Hence $F$ is central in $G$. This implies that $G$ is a Schur covering extensions of $G / F$ by $F$, so it follows that $|F|=\left|M\left(\mathrm{PSL}_{2}(4)\right)\right|=2$ and $G \cong \mathrm{SL}_{2}(5)$.

Now we deal with infinite perfect groups in the class $\mathcal{S}$.

Lemma 4.9. Let $G$ be a perfect group lying in the class $\mathcal{S}$. Then $G$ is simple if and only if its Fitting subgroup is trivial.

Proof. Let $F$ denote the Fitting subgroup of $G$. First suppose $G$ is simple. If $G=F$ then $G$ is nilpotent, a contradiction since $G$ is perfect. Therefore $F=1$. Now suppose $F=1$, and let $N$ be any proper normal subgroup of $G$. Since $G \in \mathcal{S}$, the subgroup $N$ is nilpotent, so $N \leq F$. Therefore $N=1$, and $G$ is simple.

Lemma 4.10. An infinite perfect group lying in the class $\mathcal{S}$ cannot be a Fitting group.

Proof. Let $G \in \mathcal{S}$ be an infinite perfect group, and suppose that $G$ is a Fitting group. The group $G$ cannot be minimal non-nilpotent by (see [4, Proposition 144] and [1, Corollary 1.4]), so there exists a proper non-nilpotent subgroup $H$ of $G$. Choose $x \in G \backslash H$. Since $G$ is generated by its nilpotent normal subgroups, there exists a normal subgroup $N$ of $G$ such that $N$ is nilpotent and $x \in N$. Hence $N \nsubseteq H$. Let $B$ be the last term of the derived series of $N$ which is not contained in $H$. Put $K=H B$. Then $K \in \mathcal{S}$ is locally nilpotent and non-nilpotent. Put $L=B \cap H$. Thus $L$ is normal in $K$, and the normal series $L<B<K$ can be refined to a (general) principal series of $K$. As in the proof of Theorem 3.7, all factors of this principal series which lie between $L$ and $B$ are trivial. This means $L=B$, a contradiction.

Note that the above shows that the finiteness hypothesis in Lemma 4.1 may be omitted.

Proposition 4.11. Let $G \in \mathcal{S}$ be a perfect group, and let $F$ denote its Fitting subgroup. Then $G / F$ is a non-abelian simple group.

Proof. By Lemma 4.1 we may assume that $G$ is infinite. Moreover, by Lemmas 4.9 and 4.10 we may assume that $F$ is a non-trivial proper subgroup of $G$. Clearly $F$ is infinite and contains all proper normal subgroups of $G$.

We leave it as an open problem whether or not there exist infinite perfect groups in $\mathcal{S}$ which are not simple. Note that, if such a group $G$ is locally graded and finitely generated, then $G / F$ is still locally graded (see for instance [13]), and hence it has to be finite. Therefore, by Proposition 4.4, G/F is isomorphic to $\operatorname{PSL}_{2}\left(2^{n}\right)$, where $2^{n}-1$ is a prime.

\section{References}

[1] A. O. Asar, Locally nilpotent $p$-groups whose proper subgroups are hypercentral or nilpotentby-Chernikov, J. London Math. Soc. 61 (2000), 412-422, doi:10.1112/s0024610799008479.

[2] M. J. J. Barry and M. B. Ward, Simple groups contain minimal simple groups, Publ. Mat. 41 (1997), 411-415, doi:10.5565/publmat_41297_07.

[3] Y. Berkovich, Groups of Prime Power Order, Volume 1, volume 46 of De Gruyter Expositions in Mathematics, Walter de Gruyter GmbH \& Co., Kammergericht, Berlin, 2008, https: //www. degruyter.com/view/product/178995. 
[4] C. Casolo, Groups with all subgroups subnormal, Note Mat. 28 (2008), 1-153, doi:10.1285/ i15900932v28n2supplp1.

[5] J. H. Conway, R. T. Curtis, S. P. Norton, R. A. Parker and R. A. Wilson, Atlas of Finite Groups, Oxford University Press, Eynsham, 1985, http: //brauer. maths. qmul ac . uk/Atlas/v3/.

[6] C. Delizia, H. Dietrich, P. Moravec and C. Nicotera, Groups in which every non-abelian subgroup is self-centralizing, J. Algebra 462 (2016), 23-36, doi:10.1016/j.jalgebra.2016.04.035.

[7] C. Delizia, U. Jezernik, P. Moravec and C. Nicotera, Groups in which every non-cyclic subgroup contains its centralizer, J. Algebra Appl. 13 (2014), 1350154, doi:10.1142/ s0219498813501545.

[8] C. Delizia, U. Jezernik, P. Moravec and C. Nicotera, Groups in which every non-abelian subgroup is self-normalizing, Monatsh. Math. (2017), doi:10.1007/s00605-017-1035-0.

[9] C. Delizia, U. Jezernik, P. Moravec, C. Nicotera and C. Parker, Locally finite groups in which every non-cyclic subgroup is self-centralizing, J. Pure Appl. Algebra 221 (2017), 401-410, doi:10.1016/j.jpaa.2016.06.015.

[10] L. E. Dickson, Linear Groups with an Exposition of the Galois Field Theory, B. G. Teubner, Leipzig, 1901.

[11] B. Huppert, Endliche Gruppen I, volume 134 of Die Grundlehren der mathematischen Wissenschaften, Springer-Verlag, Berlin, 1967.

[12] E. I. Khukhro, p-Automorphisms of Finite p-Groups, volume 246 of London Mathematical Society Lecture Note Series, Cambridge University Press, Cambridge, 1998, doi:10.1017/ cbo9780511526008.

[13] P. Longobardi, M. Maj and H. Smith, A note on locally graded groups, Rend. Sem. Mat. Univ. Padova 94 (1995), 275-277, http: / / www . numdam. org/item? id=RSMUP_ 1995_94_275_0.

[14] M. F. Newman and J. Wiegold, Groups with many nilpotent subgroups, Arch. Math. 15 (1964), 241-250, doi:10.1007/bf01589192.

[15] A. Y. Ol'shanskiü, Geometriya opredelyayushchikh sootnosheniŭ v gruppakh, Sovremennaya Algebra, Nauka, Moscow, 1989.

[16] D. J. S. Robinson, Finiteness Conditions and Generalized Soluble Groups, Part 1, volume 62 of Ergebnisse der Mathematik und ihrer Grenzgebiete, Springer-Verlag, Berlin, 1972.

[17] D. J. S. Robinson, Finiteness Conditions and Generalized Soluble Groups, Part 2, volume 63 of Ergebnisse der Mathematik und ihrer Grenzgebiete, Springer-Verlag, Berlin, 1972.

[18] D. J. S. Robinson, A Course in the Theory of Groups, volume 80 of Graduate Texts in Mathematics, Springer-Verlag, New York, 2nd edition, 1996, doi:10.1007/978-1-4419-8594-1.

[19] J. S. Wilson, Groups with every proper quotient finite, Proc. Cambridge Philos. Soc. 69 (1971), 373-391, doi:10.1017/s0305004100046818.

[20] R. A. Wilson, The Finite Simple Groups, volume 251 of Graduate Texts in Mathematics, Springer-Verlag, London, 2009, doi:10.1007/978-1-84800-988-2. 